\title{
Female urethral diverticulum
}

Hong-Hau Wang, Yu-Cheng Wu, Chia-Hsin Liu, Ting-Fu Su, Guo-Shu Huang, Ching-Jiunn Wu

Department of Radiology (HHW, YCW, GSH, CJW); Department of Internal Medicine (CHL) and Department of Pathology (TFS), Tri-Service General Hospital, National Defense Medical Center, Taipei, Taiwan (R.O.C.)

A 36-year-old woman presented to our department with a 6-week history of dysuria, urinary frequency and refractory urinary tract infection. Physical examinations revealed a tender cystic mass in the anterior vaginal wall. Laboratory test showed the following: leukocyte count, $13610 / \mathrm{mm}^{3}$; C-reactive protein, $2.3 \mathrm{mg} / \mathrm{dL}$. Urinalysis demonstrated microscopic pyuria (50-75 white blood cells/ high power field). Transabdominal ultrasonography disclosed a complex cystic mass on the base of the urinary bladder. Voiding cystourethrogram showed contrast material filling the urethral diverticulum (UD) that encircled the urethral lumen (Figure-1). Coronal and axial T2-weighted MR images demonstrated a circumferential high-signal intensity, fluid-

Figure 1 - Voiding cystourethrogram showed contrast material filling the urethral diverticulum (arrowheads) that encircled the urethral lumen.

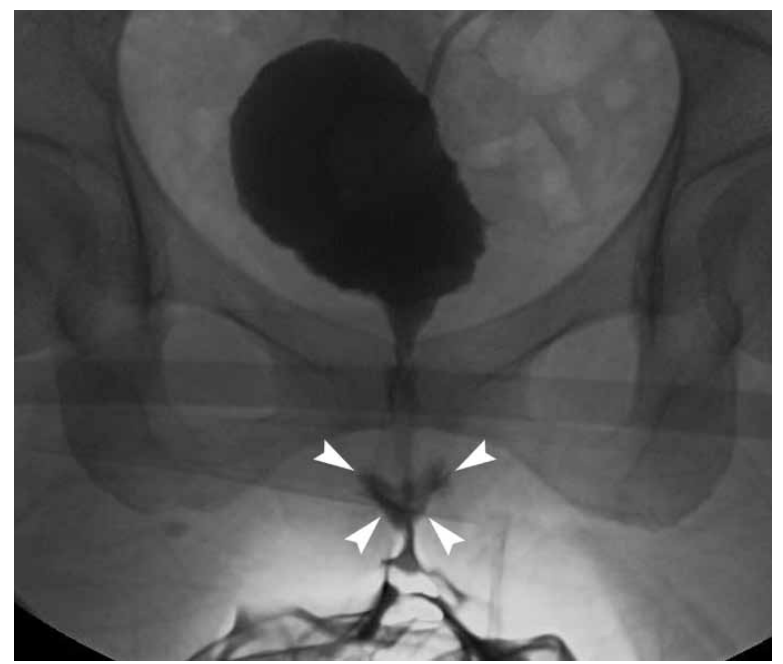

-filled lesion with fluid-debris level and confirmed the diagnosis of UD (Figure-2). Cystourethroscopy showed two orifices of the UD (Figure-3). Transvaginal diverticulectomy was performed and postoperative course was uneventful.

A UD is a focal outpouching of the urethra and usually occurs in women in the 3rd-7th decade of life, with an estimated prevalence of $0.6-6 \%$ (1). The vast majority of UDs are from acquired causes, with the most widely accepted theory involving rupture of a chronically obstructed and infected periurethral gland into the urethral lumen. Risk factors for acquired UDs include repeated infection of the periurethral glands, vaginal birth trauma, trauma from the prior vaginal or urethral procedures (1).

The classic presentation of UD has been described historically as the "three Ds": dysuria, dyspareunia, and dribbling (post-void). The most useful imaging modality for UDs is MRI (2). MRI plays an important role in the diagnosis of UDs and ideally provides the surgeon with preoperative information regarding location, number, size, configuration, and communication of the UDs. Although MRI is the best preoperative diagnostic tool for evaluating the UDs, the old standby double balloon pressure urethrography (adding sky-blue or brilliant green as staining agent) is of great value of intraoperative identification of these compound diverticular sacks and facilitating their resection (3).

Complications associated with UDs include recurrent infection, urinary incontinence, calculus formation, and development of intradiverticular neoplasm. Clinicians should be aware of the possibility of a UD in women with unexplained 
Figure 2 - (A) Coronal and (B) axial T2-weighted MR images demonstrated a circumferential high-signal intensity, fluid-filled

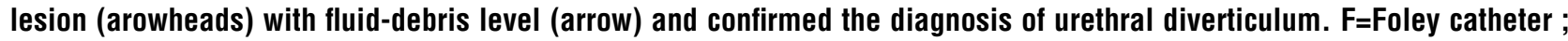
UB=Urinary bladder.

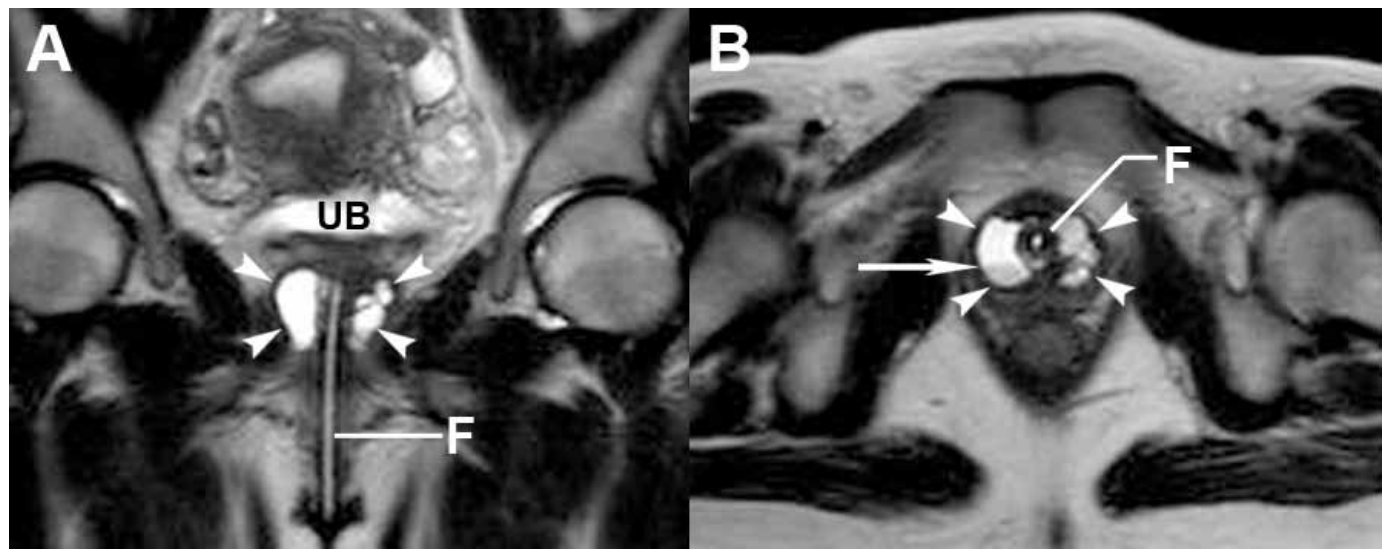

Figure 3 - Cystourethroscopy showed two orifices of the UD.

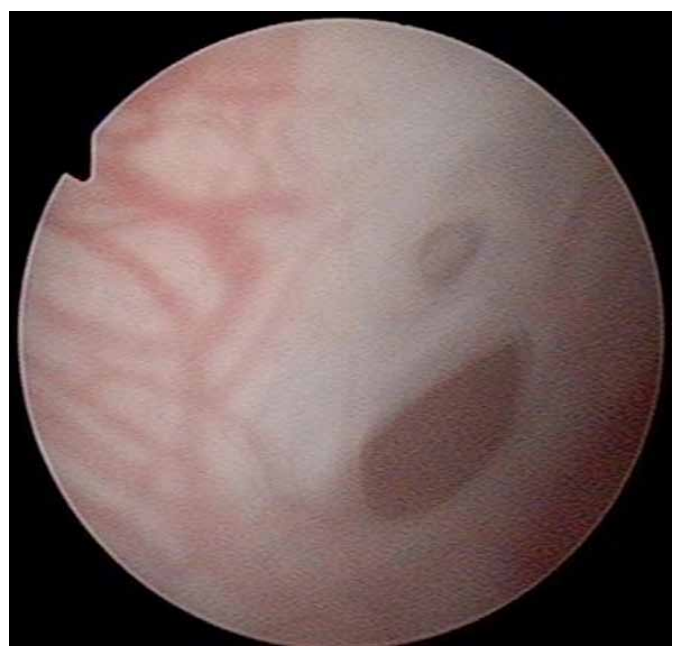

chronic or recurrent lower urinary tract symptoms. Conservative management is suited for patients with absent or mild symptoms. The definitive treatment for patients with significant symptoms caused by UDs is surgical excision (1).

\section{ABBREVIATIONS}

$$
\mathrm{UD}=\text { urethral diverticulum } .
$$

\section{REFERENCES}

1. Lee JW, Fynes MM: Female urethral diverticula. Best Pract Res Clin Obstet Gynaecol. 2005; 19: 875-93.

2. Ockrim JL, Allen DJ, Shah PJ, Greenwell TJ: A tertiary experience of urethral diverticulectomy: diagnosis, imaging and surgical outcomes. BJU Int. 2009; 103: 1550-4.

3. Lang EK, Davis HJ: Positive pressure urethrography: a roentgenographic diagnostic method for urethral diverticula in the female. Radiology. 1959; 72: 401-5.

\section{ARTICLE INFO}

Int Braz J Urol. 2013; 39: 597-8

Submitted for publication: January 05, 2013

Accepted after revision:

February 18, 2013

Correspondence address: Dr. Ching-Jiunn Wu Department of Radiology, Tri-Service General Hospital, 325, Sec. 2, Cheng-Kung Road,

Taipei, 114, Taiwan, Republic of China. Fax: +88 628 792-7245 E-mail:whh0302@gmail.com 